\title{
Component Carrier Selection and Beamforming on Carrier Aggregated Channels in Heterogeneous Networks
}

\author{
Changyin Sun ${ }^{1}$, Hongfeng Qing ${ }^{2}$, Shaopeng Wang ${ }^{2}$, Guangyue Lu ${ }^{1}$ \\ ${ }^{1}$ Department of Communication and Information Engineering, University of Xi' an Posts and Telecommunications, \\ Xi'an, China \\ ${ }^{2}$ ZTE Cooperation, Xi'an, China \\ Email: changyin_sun@163.com
}

Received June, 2013

\begin{abstract}
In this paper, component carrier selection and beamforming on carrier aggregated channels in Heterogeneous Networks are proposed. The scheme jointly selects the component carrier and precoding (i.e. beamforming) vectors with the cooperation of the other cells to deal with the interference between Macro cell and Pico cell. The component carrier selection and beamforming is achieved by optimizing the multi-cell downlink throughput. This optimization results in shutting down a subset of the component carrier in order to allow for a perfect interference removal at the receive side in the dense low power node deployment scenario. Additionally, algorithm based on Branch and Bound Method is used to reduce the search complexity of the algorithm. Simulation results show that the proposed scheme can achieve high cell-average and cell-edge throughput for the Pico cell in the Heterogeneous Networks.
\end{abstract}

Keywords: Heterogeneous Network; Inter-cell Interference Coordination; Beamforming; Carrier Aggregation

\section{Introduction}

The concept of Heterogeneous networks has attracted a lot of interests recently to optimize the performance of the network[1]. In Heterogeneous networks(HetNet), the network topology is improved by overlaying the planned network of high power Macro base stations with smaller low power Pico base stations that are distributed in an unplanned manner or simply in hotspots where a lot of traffic is generated. These deployments can improve the overall capacity and the cell edge user performance [2].

With HetNet deployment in same spectrum, users can experience severe interference. This effect is due to the often geographically random low power node deployment as well as the near-far problem arising from the imbalance in path-gains and transmission powers between the macro cell and low power nodes. 3GPP-LTE has devoted significant standardization effort towards devising inter-cell interference coordination (ICIC) schemes for minimizing interference, culminating in the so-called "enhanced" ICIC (eICIC) in LTE-Advanced.

The eICIC is one of the most important features of LTE Advanced, without it the range extension concept [3] loses its advantage and efficiency. The eICIC solutions are mainly divided into frequency domain solutions such as carrier aggregation and time domain solutions such as almost blank subframes (ABS) [2]. The main frequency domain multiplexing inter-cell interference coordination scheme used in LTE-Advanced is carrier aggregation, which basically enables a LTE-Advanced user equipment (UE) to be connected to several carriers simultaneously[2].

Carrier aggregation (CA) not only allows resource allocation across carriers but also allows scheduler based fast switching between carriers without time consuming handovers, which means that a node can schedule its control information on a carrier and its data information on another carrier. So by scheduling control and data information for both Macro and Pico layers on different component carriers, interference on control and data can be avoided. It is also possible to schedule center Pico-eNodeB(eNB) user data information on the same carrier that the Macro layer schedules its users, as the interference from the Macro layer on center Pico-eNB users can be tolerated, while Pico-eNB users in the range extension areas are still scheduled in the other carrier where the Macro-eNB users are not scheduled[4-6] .

In case of eICIC, only loose coordination among macros and picos is needed, which is advantageous from a deployment perspective. Coordinated multipoint transmission (CoMP) aims to achieve additional gains on top of eICIC by tightening the coordination among cells. For example, considering the practical constraints for joint transmission, paper [7] focuses on Coordinated scheduling/beamforming $(\mathrm{CS} / \mathrm{CB})$ based CoMP schemes in 
which the concept of resource partitioning and almost blank subframes is used firstly as an effective way of mitigating the interference between the pico cell and macro cell, and then on shared subframes, $\mathrm{CS} / \mathrm{CB}$ is applied for improved interference coordination. As a result, scheduling and beam selection gains can be achieved. However, as the number of picos grows, it becomes much harder to choose a beam good for every pico, if possible at all, so the beam selection gain will vanish. Paper [8] also employ multiple antennas in two-tier femtocell networks to provide additional degrees of freedom that can be used to help coordinate the cross-tier interference, it propose a beamforming codebook restriction strategy. Although restricting the beamforming codebook increases the quantization error for the macrocell users, proportional fair scheduler compensate for the increased quantization error by exploiting the channel selection diversity gain and the multiuser diversity gain. As the number of femtocell grows, the opportunistic channel selection strategy will loose the limited additional degrees of freedom.

In this paper, component carrier selection and Beamforming on carrier aggregated channels in Heterogeneous Networks is addressed. The Heterogeneous Networks consists of complementing the Macro layer with low power nodes such as Pico base stations, and solution such as Range Extension is assumed to extend the coverage area of the Pico nodes. The scheme jointly selects the component carrier and precoding (i.e. beamforming) vectors with the cooperation of the other cells to deal with the interference between Macro cell and Pico cell. The component carrier and beamforming selection is optimized not only to exploit additional degrees of freedom provided by multiple antennas and component carriers, but also to restore the feasibility of the $\mathrm{CS} / \mathrm{CB}$ when the number of low power nodes grows. As a result, the design will shut down a subset of the component carrier in order to allow for a perfect interference removal at the receive side in the large dense low power node deployment scenario. Additionally, algorithm based on Branch and Bound Method is used to reduce the search complexity of the algorithm. The proposed approach is confirmed by simulation results compared with the performance of the baseline approach.

\section{System Model}

We consider Hetnet network with the following features: 1) a certain number of Pico-eNBs are deployed throughout one Macro cell layout; 2) The Pico-eNBs are randomly distributed; 3) The users are randomly distributed throughout the cell area.

Now consider the downlink transmission with M users and $\mathrm{K}$ carriers in the network. The BSs and the users are assumed to have $\mathrm{N}$ transmit antenna and one receive antenna, respectively. For simplicity, the transmit power is kept the same in each carrier per cell $q$, i.e. $P_{q}$. Let the binary matrix $\mathbf{A}=\left\{\mathrm{a}_{k, m} \mid \mathrm{a}_{k, m} \subset\{0,1\}\right\}_{K \times M}$ describes the carrier selection among the users, where $a_{k, m}=1$ denotes that carrier $\mathrm{k}$ is assigned to user $\mathrm{m}$, otherwise, $a_{k, m}=0$.

Now, denote by $S_{k, m, i}=\mathbf{h}_{k, m, i} \mathbf{b}_{k, i}$ the channel power gain to the selected mobile user $\mathrm{m}$ in cell $\mathrm{q}$ from the cell $\mathrm{i}$ base station, in resource slot $t$, where

$$
\mathbf{h}_{k, m, i}=\left[h_{k, m, i}^{1} h_{k, m, i}^{2}, \cdots, h_{k, m, i}^{N}\right]
$$

denotes the channel vector of the user $m$ in cell $q$ from the cell i base station, $\mathbf{b}_{k, i} \in \mathbf{C}^{N \times 1}$ is the beamforming vectors used to map the user in cell $\mathrm{i}$ data symbols to the transmit signals. The channel gains are assumed to be constant over each such resource slot, i.e., we have a block fading scenario. Note that the gain $S_{k, m, q}$ corresponds to the desired communication link, whereas the gains $S_{k, m, i}$ for $i \neq q$ correspond to the unwanted interference links. Assuming the transmitted symbols to be independent random variables with zero mean and a variance $P_{q}$, the signal to noise-plus-interference ratio (SINR) for each user is given by:

$$
\operatorname{SINR}_{k, m, q}=\frac{P_{q}\left|\mathbf{h}_{k, m, q} \mathbf{b}_{k, q}\right|^{2}}{\sum_{i=1, i \neq q}^{N} a_{k, i} P_{i}\left|\mathbf{h}_{k, m, i} \mathbf{b}_{k, i}\right|^{2}+\sigma_{q}^{2}}
$$

where $\sigma$ is the variance of the independent zero-mean AWGN.

Then the achievable rate for user $\mathrm{m}$ is given by:

$$
R_{m, q}=\sum_{k=1}^{K} \log _{2}\left(1+\operatorname{SINR}_{k, m, q}\right)=\sum_{k=1}^{K} R_{k, m, q}
$$

Assume at time slot $t$, only one user $m \in S(q)$ is scheduled at each base-station q, so we will not distinguish cell q and the user served by cell q for simplicity, then from (1) and (2) the total achievable throughput (sum rate) $R=\sum_{q=1}^{Q} R_{m, q}$ is then found as

$$
R=\sum_{q=1}^{Q} \sum_{k=1}^{K} \log _{2}\left(1+\frac{a_{k, q} P_{q}\left|\mathbf{h}_{k, q, q} \mathbf{b}_{k, q}\right|^{2}}{\sum_{i=1, i \neq q}^{N} a_{k, i} P_{i}\left|\mathbf{h}_{k, q, i} \mathbf{b}_{k, i}\right|^{2}+\sigma_{q}^{2}}\right)
$$

The component carrier selection and interference coordination problems are to find the matrix $\mathbf{A}$ and $\mathbf{b}_{k, q}$ such that the objective function is optimized. Assuming the goal is to achieve the highest system throughput while ensuring proportional fairness among different users, the following utility function needs to be maximized:

$$
\begin{gathered}
R=\sum_{q=1}^{Q} \sum_{k=1}^{K} \alpha_{q} R_{k, q} \\
\text { s.t.1) } \sum_{k=1}^{K} a_{k, q} \leq C_{q} \\
\text { 2) }\left|\mathbf{b}_{k, q}\right|=1
\end{gathered}
$$


The constrained problem (4) is a mixed binary-nonconvex problem. To find the global optimum, one has to exhaustively search through all possible $\mathbf{b}_{k, i}$ (real values) and $a_{k, q}$ (binary values). Here, we shall first decompose it into the $\mathrm{K}$ independent per-carrier objective function, then adopt the method of alternatingly optimizing antenna vectors and the component carrier selection.

The Lagrangian of problem (4), dualized with respect to the constraint 1) is defined as:

$$
J=\sum_{k=1}^{K}\left\{\sum_{q=1} \alpha_{q} R_{k, q}-\sum_{q} \lambda_{q} a_{k, q}\right\}+\sum_{q} \lambda_{q} C_{q}
$$

This Lagrangian can be decomposed into the $\mathrm{K}$ independent per-carrier objective function. For a particular $\lambda_{q}$ the optimization problem (5) can be solved in a per-carrier fashion:

$$
\begin{array}{cl}
\left\{\mathbf{B}_{k}, \mathbf{a}_{k}\right\}^{\text {opt }} & =\arg \min \left\{-J_{k}\right\} \\
\text { s.t. } & \text { 1) }\left|\mathbf{b}_{k, q}\right|=1 \\
& \text { 2) } \lambda_{q} \geq 0 \\
& \text { 3) } a_{k, q} \in\{0,1\}
\end{array}
$$

where $-J_{k}=\sum \alpha_{q} R_{k, q}-\sum \lambda_{q} a_{k, q}$.

To solve (4) by (6), $\lambda_{q}$ should be tuned to enforce the constraints as in [9], where an efficient Lagrange multiplier search procedure is presented. This procedure for the Lagrange multipliers is assumed in the following update formula:

$$
\begin{gathered}
\lambda_{q}^{t+1}=\lambda_{q}^{t}+\mu_{\lambda}\left(C_{q}-\sum_{k=1}^{K} a_{k, q}\right)^{+} \\
q=1,2, \cdots Q,
\end{gathered}
$$

where $(x)^{+}$means $\max (0, x), \mathrm{t}$ is the iteration number, $\mu_{\lambda}$ is a step size parameter and $C_{q}$ is the number of carrier selected corresponding to the Lagrange multipliers at hand.

Assume $\lambda_{q}$ is fixed and $a_{k, q}$ is selected on carrier $\mathrm{k}$, the transmit beamforming vector $\mathbf{b}_{\mathbf{q}}$ of cell $\mathrm{q}$ in carrier $\mathrm{k}$ which maximizes the sum rate in (6) is given by the following dominant eigenvector problem [10]:

$$
\left(\mathbf{E}_{\mathbf{q}}+\sum_{i=1, i \neq q}^{Q} \lambda_{i, q} \mathbf{A}_{\mathbf{i}, \mathbf{q}}\right) \mathbf{b}_{\mathbf{q}}=\mu_{b} \mathbf{b}_{\mathbf{q}}
$$

where $\mathbf{E}_{\mathbf{q}}=\mathbf{h}_{\mathbf{q}, \mathbf{q}}^{\mathrm{H}} \mathbf{h}_{\mathbf{q}, \mathbf{q}}, \quad \mathbf{A}_{\mathbf{i}, \mathbf{q}}=\mathbf{h}_{\mathbf{i}, \mathbf{h}}^{\mathrm{H}} \mathbf{h}_{\mathbf{i}, \mathbf{q}}$ and the real values $\lambda_{i, q}$ is defined as following:

$$
\lambda_{i, q}=-\frac{\mathbf{I}_{\mathbf{q}}+\left|\mathbf{h}_{\mathbf{q}, \mathbf{q}} \mathbf{b}_{\mathbf{q}}\right|^{2} P_{q}+\sigma_{q}^{2}}{\mathbf{I}_{\mathbf{i}}+\left|\mathbf{h}_{\mathbf{i}, \mathbf{i}} \mathbf{b}_{\mathbf{i}}\right|^{2} P_{i}+\sigma_{q}^{2}} \times \frac{\left|\mathbf{h}_{\mathbf{i}, \mathbf{i}} \mathbf{b}_{\mathbf{i}}\right|^{2} P_{i}}{\mathbf{I}_{\mathbf{i}}+\sigma_{q}^{2}}
$$

And $\mathbf{I}_{\mathbf{q}}$ is the received interference power of user q:

$$
\mathbf{I}_{\mathbf{q}}=\sum_{i=1, i \neq q}^{Q}\left|\mathbf{h}_{\mathbf{q}, \mathbf{i}} \mathbf{b}_{\mathbf{i}}\right|^{2} P_{i}
$$

The optimum point $\mathbf{b}_{q}^{*}$ are the eigenvector corresponding to the largest (resp. smallest) eigenvalue of (8).

With fixed $\mathbf{b}_{k, i}=\mathbf{b}_{q}^{*}$ we denote $\bar{h}_{k, q, q}=\left|h_{k, q, q} b_{k, q}\right|^{2}$, $\bar{h}_{k, q, i}=\left|h_{k, q, i} b_{q, i}\right|^{2} \quad$ Thus, one can get $a_{k, q}$ from (6) with :

$-J_{k}=\sum_{q=1}^{Q} \sum_{k=1}^{K} \log _{2}\left(1+\frac{a_{k, q} P_{q}\left|\bar{h}_{k, q, q}\right|^{2}}{\sum_{i=1, i \neq q}^{N} a_{k, i} P_{i}\left|\bar{h}_{k, q, i}\right|^{2}+N_{q}^{2}}\right)-\sum \lambda_{q} a_{k, q}$

\subsection{Linear Convex Relaxation Approach}

Unfortunately, for given Lagrange multipliers, these decoupled optimization problems (11) are themselves difficult nonconvex problems. So a novel low-complexity optimization algorithm is presented. The algorithm is based on a relaxation of the nonconvex per-carrier optimization problem (11) leading to a more direct and conceptually simple procedure.

The convex relaxation starts with rewriting the objective function of (11) in the following form:

$$
\begin{aligned}
-J_{k}= & -\sum_{q}\left\{\log _{2}\left(\sum_{i=1}^{Q} a_{k, i} P_{i}\left|\bar{h}_{k, q, i}\right|^{2}+\sigma_{q}^{2}\right)+\lambda_{q} a_{k, q}\right\} \\
& +\sum_{q} \log _{2}\left(\sum_{i=1, i \neq q}^{Q} a_{k, i} P_{i}\left|\bar{h}_{k, q, i}\right|^{2}+\sigma_{q}^{2}\right)
\end{aligned}
$$

which consists of a convex part A (the first part) and a concave part B. This objective function is a difference of convex (d.c.) functions which is known to correspond to a hard optimization problem [9]. The crucial step is now to relax the nonconvex part B by hyperplane overestimators, leading to the following relaxed objective:

$$
-J_{k}^{\text {rel }}=A+\sum_{q}\left(\sum_{i=1, i \neq q}^{Q} u_{k, i} a_{k, i}+v_{k, i}\right)
$$

where $\left(\sum_{i=1, i \neq q}^{Q} u_{k, i} a_{k, i}+v_{k, i}\right)$

$$
\geq \log _{2}\left(\sum_{i=1, i \neq q}^{Q} a_{k, i} P_{i}\left|\bar{h}_{k, i}\right|^{2}+\sigma_{q}^{2}\right)
$$

This is tight with equality at approximation point $\bar{a}_{k, i}$. The obtained relaxed objective function is a convex function. The constraints are also convex leading to a convex optimization problem which can be solved efficiently.

The solution of this convex relaxation forms an upper bound for the global minimum. Using the obtained upper bound as a new point of approximation (see algorithm 1) it can be proven that the sequence of relaxations produces a monotonically decreasing objective value and will always converge. The proof is trivial and omitted due to space limitations. Upon convergence it can be proven that the obtained solution is a local optimum. Although there is no theoretical proof for global optimality, simulation results are very promising showing global optimality for very different scenarios.

\subsection{Branch and Bound Method}

The binary convex problems (11) are solved by the Branch and bound. Branch and bound [11] is a general 
technique for finding optimal solutions of various optimization problems. A branch and bound procedure has two ingredients. The first ingredient is a smart way of covering the feasible region by several smaller subregions. This leads to a branching operation. The second ingredient is bounding, which is a way of finding upper and lower bounds for the optimal solution within a feasible subregion. The core of the approach is the simple observation that (for a maximization task) if the upper bound of a subregion $\mathrm{A}$ is smaller than the lower bound for some other subregion B, then subregion A may be safely discarded from the search.

To efficiently solve this problem by branch and bound method, the problem is convex relaxed as:

$$
\left\{\mathbf{a}_{\mathbf{k}}\right\}^{\text {opt }}=\arg \min \left\{-J_{k}^{\text {rel }}\right\}
$$

Subject to:

$$
\begin{gathered}
\lambda_{q} \geq 0 \\
a_{k, i} \in[0,1]
\end{gathered}
$$

Which is convex with continues variable $\mathbf{a}_{k}$, if we denote $L_{1}$ as its optimal value, and the optimal value of the original problem is denoted $p^{*}$, then $L_{1}$ is a lower bound on the optimal value of (11).

An upper bound (denoted $U_{1}$ ) on $p^{*}$ can be fund by several ways, one more sophisticated method: round each binary variable $\mathbf{a}_{k}$ then we'll obtain this upper bound. If $U_{1}-L_{1} \leq \varepsilon$, we can quit.

In the branching operation, we pick any index $\{q\}$, and form two subproblems: the first problem and the second problem. The first and the second problem is the relaxed problems with $a_{k, q}=0$ and $a_{k, q}=1$ respectively. Solving the first and the second problem, we obtain the \{lower, upper $\}$ bounds $\{\tilde{L}, \tilde{U}\}$ and $\{\bar{L}, \bar{U}\}$ for $a_{m, k}=0$ and $a_{m, k}=1$ respectively, thus the new bounds on $p^{*}$ :

$$
\left.L_{2}=\min \{\tilde{L}, \bar{L}\} \leq p^{*} \leq \min \{\tilde{U}, \bar{U})=U_{2}\right\}
$$

The Branch and bound algorithm continue to form binary tree by splitting, relaxing, calculating bounds on subproblems. The common strategy to select the leaf node for further split operation is to pick a node with smallest L, while that to select the variable for further split operation is either the 'least ambivalent' or the 'most ambivalent' way. The 'least ambivalent' way will choose $\{\mathrm{q}\}$ for which $q^{*}=\{0,1\}$, with largest Lagrange multiplier, while the 'most ambivalent' way will choose $\{\mathrm{q}\}$ for which $\left|q^{*}-1 / 2\right|$ is minimum.

Table 1. Algotithm1.

\begin{tabular}{l} 
Algorithm 1 Successive linear convex relaxation for CC k \\
\hline 1: Repeat 1 \\
2: For $\mathrm{i}=1$ to $\mathrm{Q}$. \\
3: tighten: compute $u_{k, i}(l) v_{k, i}(l)$ \\
4: End For. \\
5: minimize: solve problem (12) with Relaxed $\left(J_{k}^{\text {rel }}\right)$ \\
6:End Loop until convergence
\end{tabular}

Table 2. Algorithm 2.

Algorithm 2 Branch and bound method

Branching:

1: choose one of the carrier selection index $\{\mathrm{q}\}$;

2: solve two convex relaxed problems (11): set $a_{k, q}=0$ and

$a_{k, q}=1$ respectively.

Bounding:

3: take the optimal value of the two convex relaxed problems as the lower bounds $=\{\tilde{L}, \bar{L}\}$;

4: take the solution of the two convex relaxed problems as the upper bounds $=\{\tilde{U}, \bar{U}\}$ when round all the relaxed binary variables to 0 or 1

5: take minimum of the lower bounds as the current lower bound $L_{2}=\min \{\tilde{L}, \bar{L}\}$ on the optimal value of the optimization problem;

6: take minimum of the upper bounds as the current upper bound $U_{2}=\min \{\tilde{U}, \bar{U})$ on the optimal value of the optimization problem;

Pruning:

7: If $U_{2}-L_{2} \leq \varepsilon$ then quit the algorithm.

8:else select the leaf node for further split operation :select the sub-tree (sub-problem) with the smallest lower bound to be split; 9: end if

10: select the variable for further split operation :select another index $\{\mathrm{q}\}$ which has not been so far used while meet the 'most ambivalent' or the 'most ambivalent' requirement;

11 : go to step 2.

\section{Simulation Results}

In this section, simulation results are presented to evaluate the performances of the proposed scheme in a carrier aggregation system. For comparison purpose, the performances of the network with all the carriers fully reused between macro and pico station are also given. For simplicity, we only consider one cell network which contains 1 Macro-eNB and 2 Pico-eNBs. The radius of Macro cell is $1000 \mathrm{~m}$. And the total number of users is 40 . The range extension threshold is $10 \mathrm{~dB}$. Detailed simulation parameters including channel model and system assumptions are summarized in Table 3. Most of them are set according to the LTE-A simulation assumptions.

First, equal cell specific weight in the system sum rate is used, and none uniform user distribution across the macro cell is assumed, in this case, the users are dropped at the dense area which overlay both the pico cells and is about $12 \%$ of the Macro cell area. The cumulative distribution function (CDF) performances of the networks with the new joint carrier selection and beamforming scheme and frequency reuse 1 with beamforming scheme are compared in Figure 1. Compared to the networks where carriers are fully reused ('Reuse 1') between Macro cell and pico cell, the throughput performance of the macro cell with the proposed interference coordination scheme is significantly improved when equal cell specific weight is applied. On the contrary, the throughput performance of the Pico cell with the proposed scheme is a little improved compared with that of the 
baseline scheme. Moreover, in order to clearly verify the effects of the proposed scheme, we also present the performance of the network throughput. Compared to the baseline scheme where carriers are fully reused ('Reuse 1') between Macro cell and pico cell, the system through put performance with the proposed interference coordination scheme is significantly improved. In this case, we see gains beyond simple beamforming coordination gain.

Table 3. Simulation Assumption.

\begin{tabular}{lc}
\hline \multicolumn{1}{c}{ Parameters } & Value \\
\hline Carrier bandwidth & $10 \mathrm{MHz}$ \\
Number of Carriers & 2 \\
Path loss model Pico (dB) & $140.7+36.7 \log 10(\mathrm{R}), \mathrm{R}$ in km \\
Path loss model Macro (dB) & $128.1+37.6 \log 10(\mathrm{R}), \mathrm{R}$ in km \\
Shadowing standard devition (dB) & 8 \\
Transmit power Macro (dBm) & 43 \\
Transmit power Pico (dBm) & 20 \\
Number of Tx Antenna & 2 \\
Number of Re Antenna & 1 \\
\hline
\end{tabular}
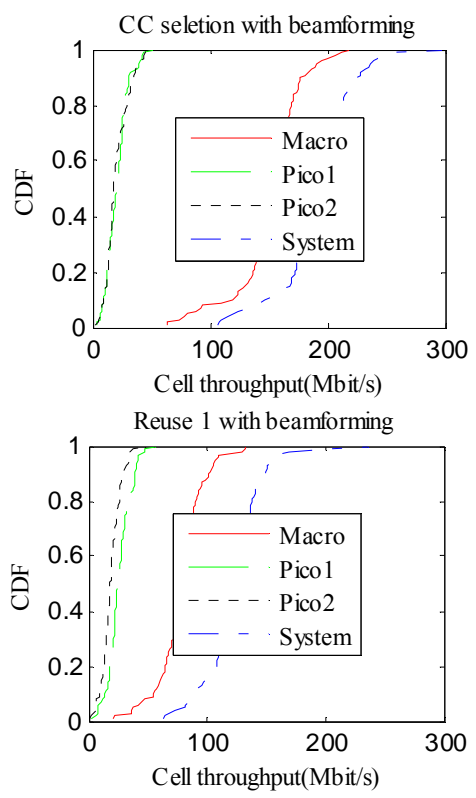

Figure 1. Performances with equal weight.

Next, the CDF performances of the networks with none uniform user distribution and with cell specific weight $\left\{\alpha_{M}, \alpha_{P 1}, \alpha_{P 2}\right\}=\{1 / 5,2 / 5,2 / 5\}$ are compared in Figure 2. Compared with the uniform cells weight $\{1,1,1\}$ in Figure 1, the throughput performance of the Pico cell with the proposed interference coordination scheme is significantly improved when cell specific weight is applied. On the contrary, the throughput performance of the Macro cell is a little improvment com- pared with that of the reuse one scheme.

In general we see there is both cell and system performance improvement in throughput in using joint carrier selection and beamforming for interference coordination over the baseline scheme in both the scenarios. The results suggest that the interference coordination not only exploit additional degrees of freedom provided by multiple antennas and component carriers, but also to restore the feasibility of the $\mathrm{CS} / \mathrm{CB}$ when the number of low power nodes grows, which is achieved by shutting down a subset of the component carrier in order to allow for a perfect interference removal at the receive side in the large dense low power node deployment scenario. More over, with different cell specific weight in sum rate, further load offset effect is observed beyond simple range extension scheme.

\section{Conclusions}

In this paper, we consider joint component carrier selection and beamforming for carrier aggregation system in Heterogeneous Networks. In the proposed scheme, $\mathrm{CS} / \mathrm{CB}$ is firstly applied for mitigating the interference between the pico cell and macro cell, then component carrier is selected to restore the feasibility of $\mathrm{CB}$ in the
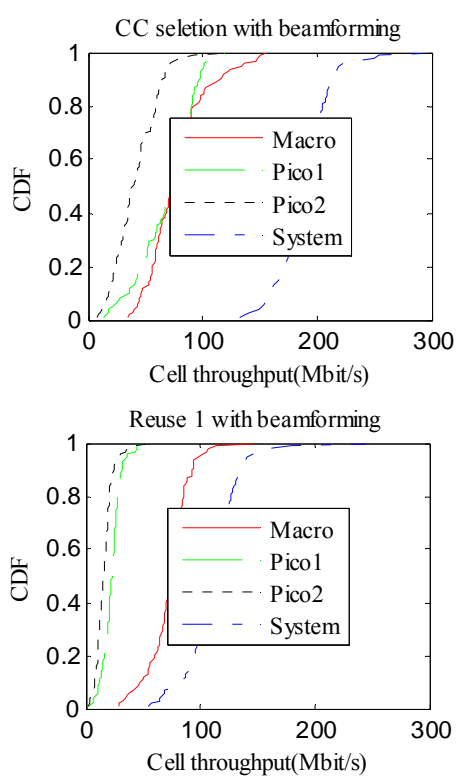

Figure 2. Performances with un-equal weight.

dense low power node scenario. The component carrier and beamforming selection is optimized not only to exploit additional degrees of freedom provided by multiple antennas and component carriers, but also to restore the feasibility of the CS/CB when the number of low power nodes grows. As a result, the design will shut down a subset of the component carrier in order to allow for a perfect interference removal at the receive side in the 
large dense low power node deployment scenario. Additionally, algorithm based on Branch and Bound Method is used to reduce the search complexity of the algorithm. The proposed approach is confirmed by simulation results compared with the performance of the baseline approach.

\section{Acknowledgements}

This work was supported in part by National Natural Science Foundation of China (61102047, 61271276); Key project (2012ZX03001025) of China, Natural Science Foundation of Education department of Shaanxi Province(2013JK1045); Foundation of ZTE Forum.

\section{REFERENCES}

[1] Qualcomm Incorporated, LTE advanced: heterogeneous networks, Feb. 2010. Availalbe:

http://www.qualcomm.co.kr/documents/lteadvanced-heter ogeneous-networks.

[2] E. Dahlman, S. Parkvall and J. Skold, "4G: LTE/LTE-advanced for Mobile Broadband," Academic Press, 2011.

[3] 3GPP Tdoc R4-083018, Qualcomm Europe, Range Expansion for Efficient Support of Heterogeneous Networks, Aug., 2008.

[4] Y. Wang, K. I. Pedersen, T. B. Sorensen and P. E. Mogensen, "Carrier Load Balancing and Packet Scheduling for Multi-carrier Systems," IEEE Transactions Wireless
Communications, Vol. 9, 2010, pp. 1780-1789. doi:10.1109/TWC.2010.05.091310

[5] Y. Yan, A. X. Li and X. Y. Gao, "Hidetoshi Kayama, A New Autonomous Component Carrier Selection Scheme for Home eNB in LTE-A System," in Proceeding IEEE VTC, 2011 Spring, May 2011.

[6] K. I. Pedersen, F. Frederiksen, C. Rosa and H. Nguyen, Carrier Aggregation for LTE-advanced: Functionality and Performance Aspects, IEEE Communications Magazine, Vol. 49, No. 6, 2011, pp. 89-95. doi:10.1109/MCOM.2011.5783991

[7] A. Barbieri, P. Gaal, S. Geirhofer, T. Ji, D. Malladi, Y. Wei and F. Xue, "Coordinated Downlink Multi-point Communications in Heterogeneous Cellular Networks," Information Theory and Applications Workshop (ITA), 2012, pp. 7-16.

[8] W. Seo, S. Choi and D. Hong, "A Beamforming Codebook Restriction for Cross-Tier Interference Coordination in Two-Tier Femtocell Networks," IEEE Transactions on Vehicular Technology, Vol. 60, No. 4, pp. 1651-166.

[9] W. Yu and R. Lui, "Dual Methods for Nonconvex Spectrum Optimization of Multicarrier Systems, IEEE Transactions Communications, Vol. 54, No. 7, 2006, pp. 1310-1322. doi:10.1109/TCOMM.2006.877962

[10] K. M. Ho Zuleita, M. Kaynia and D. Gesbert, Distributed Power Control and Beamforming on MIMO Interference Channels, Wireless Conference (EW), European, April 2010, pp. 654-660.

[11] P. M. Pardalos and H. E. Romeijn, Handbook of Global Optimization Volume 2 (Nonconvex Optimization and Its Applications), 1 Edition, Springer, 2002. 